\title{
Téoros
}

Revue de recherche en tourisme

\section{Tourisme contre développement : thèses et antithèses}

\section{Jean Stafford}

Volume 13, numéro 2, été 1994

Regards sur le tourisme dans les pays en développement

URI : https://id.erudit.org/iderudit/1077755ar

DOI : https://doi.org/10.7202/1077755ar

Aller au sommaire du numéro

Éditeur(s)

Université du Québec à Montréal

ISSN

0712-8657 (imprimé)

1923-2705 (numérique)

Découvrir la revue

Citer cet article

Stafford, J. (1994). Tourisme contre développement : thèses et antithèses.

Téoros, 13(2), 3-7. https://doi.org/10.7202/1077755ar d'utilisation que vous pouvez consulter en ligne.

https://apropos.erudit.org/fr/usagers/politique-dutilisation/ 


\section{Tourisme contre développement : thèses et antithèses}

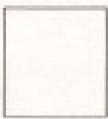

On peut sedemander quels liens il peut $y$ avoir entre le tourisme et les pays en développement: de prime abord on serait tenté de dire qu'iln'y en a aucun! Tourisme et pays en développement sont des termes qui ne vont pas trè̀ bien ensemble. Le tourisme est un pur produit de la société post-industrielle; les pays en développement font partied'un autre univers! Nos perceptions de ces pays sont biaisées par notrevision du monde limitée à l'Occident, à son histoire et à ses valeurs. Le tourisme dans les pays en développement ressemble à une machine de guerre, cheval de Troie, juxtaposition de niveaux culturels qui ne fusionnent jamais. Cette forme de tourisme repose sur des mythes, des idées reçues et des paradoxes qui nous empêchent d'avoir une compréhension plus exacte des choses.

Le tourisme est un pur produit des sociétếs industrielles avancếes

Au plan socioculturel, le tourisme tel que vécu en Occident et tel qu'il existe dans les pays en développement sont deux univers aux antipodes. Le tourisme est une industrie de riches; il correspond à des besoins difficilement justifiables en dehors des sociétés occidentales. Dans ces pays, il est la suite logique des différentes conquêtes de l'homme sur un monde hostile: après la nourriture, le logement, la santé, l'éducation, le travail et les loisirs quotidiens, le tourisme est le couronnement, l'apothéose d'un certain mode de vie.

Le tourisme est devenu, dans le monde oocidental, la fenêtre et le miroir des sociétés postmodernes orientées vers la recherche du towjours phus. Dans cette fin de siècle triomphaliste, pour les sociétés postmodernes, lemonde ressembleà une grande échelle où les pays du Nord seraient au sommet et les pays du Sud quelque part sur les barreaux inferieurs. Dans ce contexte, le seul objectif raisonnable paraît être, pour ces pays, de vouloir accéder, d'une façon ou

Monsieur Jean Stafford est professeur au département d'etudes urbaines et touristiques a I'Université du Québec à Montréal.
Les pays du Nord ont des perceptions unidimensionnelles et linéaires des pays du Sud; ces perceptions *renforcent l'idée que "ces pays" sont des réalités provisoires, sans essence propre, qui n'ont rien à nous apprendre sur eux-mêmes ou sur nous, puisqu'ils sont censéspasser fatalementau "stade supérieur ${ }^{* \prime}{ }^{(1)}$. Bizarrement, le tourisme est présenté comme le meilleur *passeport pour le développement ${ }^{(2)}$ alors qu'il ne devrait apparaître (selon la vision linéaire) qu'à la toute fin de la chaîne des besoins fondamentaux.

\section{Des trois mondes aux deux univers du tourisme}

Dans le monde occidental, le tourisme est le produit d'une longue gestation, d'interactions constantes entrel imaginaire du voyage, l'industrie touristique et les lieux à visiter. La machinerie ${ }^{9}$ du tourisme fonctionne à partir de trois mondes: trois structures superposées (voir le schéma I).

Dans ce système, l'industrie touristique, prise dans son ensemble, joue un rôle moteur: elle doit traduite dans les termes de l'imagerie culturelle les endroits à visiter et inversement façonner ces destinations en fonction del'imaginaire collectif. L'imaginaire devient demande, la demande se transforme en circuits, en modes de transports, en hébergement, en restauration, en animation, etc. D'un niveau de rationalisation à un autre, l'imaginaire s'émousse, le désir seperden un découpage rationnel du temps et de l'espace.

Pour les paysen développement, le schéma deviendra plus complexe : d'autres structures se greffent aux premières. Dans le premier schéma, nous avions trois éléments:

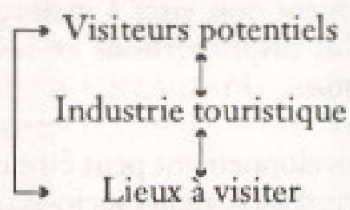

Maintenant, dans les pays en développement, nous aurons une structure indiquée au schéma II. 
Nous auronsici un deuxième niveau écrantraducteur des desiderata des uns et des autres. Tout sera en double: les systèmes organisationnels, les valeurs, les choses elles-mêmes auront des usages différents.

Déjà, dans les pays occidentaux, «la société locale, l'accumulation des signes et des symboles sur son territoire, les hommes mêmes qui y vivent n'existent qu'inventés par les schémas culturels qui organisent le regard touristiquess ${ }^{(*)}$. Dans les pays en déweloppement, ces schémas culturels subissent une double traduction; il peut même y avoir une triple traduction: imaginons un guide algérien faisant visiter un village berbère à des Occidentaux. Le tourisme, qui est déjà simulacre et thêturalité, prend dans ces pays une profondeur insoupçonnée!

\section{Développement et sous-développement}

Dans l'histoire, la croissance et le développement économiques sont des idées relativement nouvelles. Les sociétés traditionnelles admettaientquel'accumulation économique était cyclique et pouvait donc avoir des hauts et des bas. Dans les sociétés industrielles, la croissance devient une nécessité; cette croissance se fera par la modernisation des équipements et des méthodes de gestion. Parallèlement, on cherchera à conquérir denouveaux marchés et à rationaliser au maximum les processus de production. C'est à partir de ce modèle que sera défini le sous-développement.

Pour la théorie économique classique, la croissance résulte d'un savant dosage entre les biens de production et les biens de consommation ${ }^{(5)}$. Certains auteurs valorisent une croissance équilibrée par le développement simultané de diverses branches industrielles. D'autresauteurs préconisent une croissance dééquilibrée; ils perçoivent la croissance comme des séquences de déséquilibres. Pour le plus célèbre d'entre eux, Albert Hirschman: «Pour que l'économie continue de progresser, la politique de développement doit viser à maintenir les tensions, les disproportions et les déséquilibres ${ }^{(6)}$.

Le sous-développement peut être mesuré par une série d'indicateurs socio-économiques?:

- revenus par tête de moins de 4000 \$;

- économie dualiste;
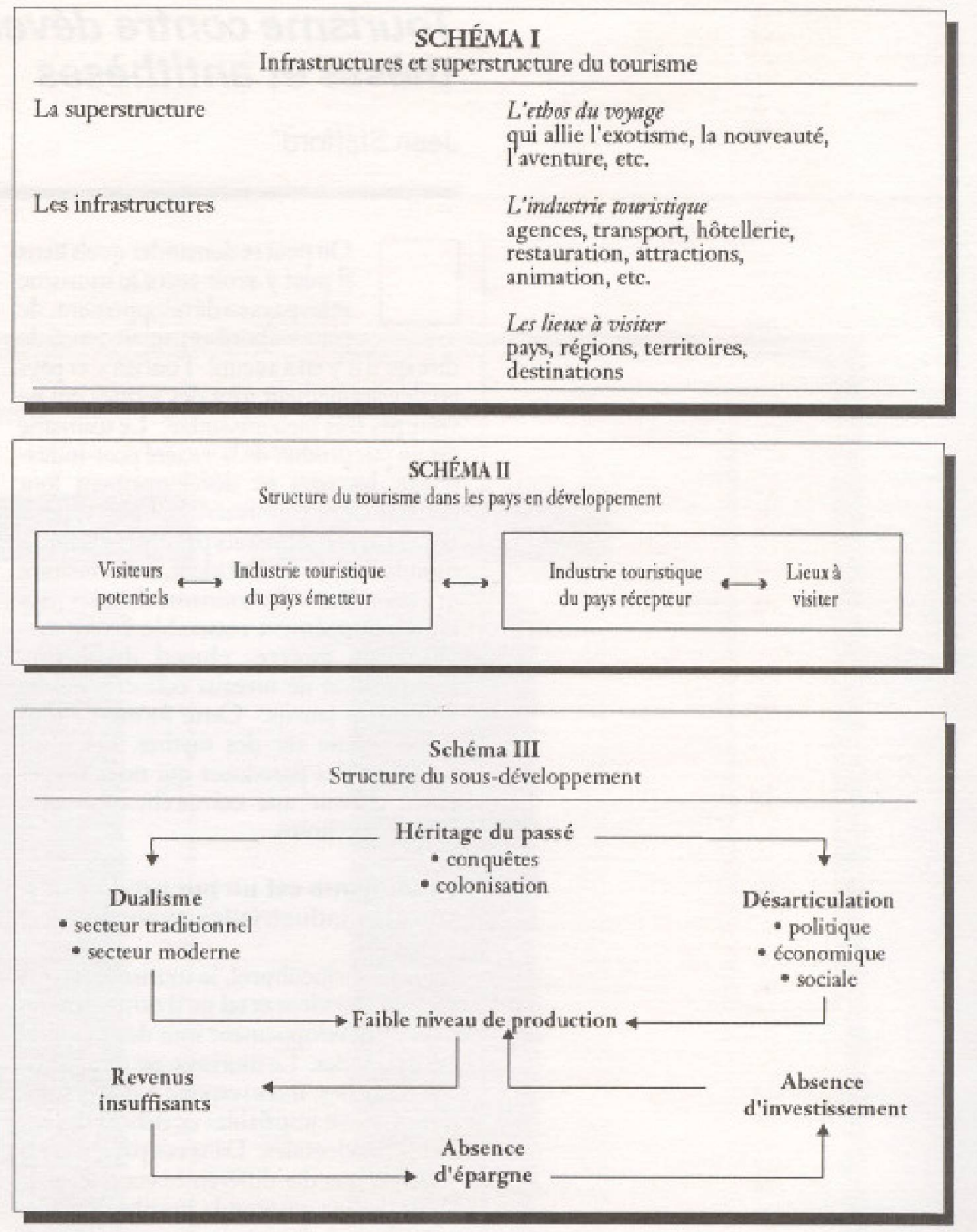

TABLEAU 1

Les parts de marché des arrivées des touristes internationaux dans les diverses régions du monde en $1962,1972,1982$ et 1992 (en \%)

\begin{tabular}{lrrrr}
\hline Régions & 1962 & 1972 & 1982 & 1992 \\
\hline Europe & 71,8 & 75,4 & 68,4 & 60,3 \\
Amériques & 23,2 & 18,2 & 18,0 & 21,0 \\
Afrique & 1,2 & 1,4 & 2,3 & 3,6 \\
Asie* & 1,8 & 3,2 & 8,9 & 13,4 \\
Moyen-Orient & 2,0 & 1,8 & 2,4 & 1,7 \\
\hline Total & 100,0 & 100,0 & 100,0 & 100,0 \\
\hline
\end{tabular}

Source: OMT.

* Il faut noter que la plus grande part des arrivées des touristes internationaux en Asie ne touchent que quelques pays: Japon, Corée du Sud, Hong Kong. 
- faiblesse de l'investissement et de la productivité;

- répartition très inégale des revenus;

- niveau très bas au plan de l'éducation et de la santé;

- forts taux de chômage;

- économie souterraine;

- etc

A partir de ces indicateurs, on distingue cinq grands groupes de pays :

1- pays en développement;

2- nouveaux pays industriels;

3- pays de l'Est;

4- pays pétroliers;

5- pays industrialisés.

Ces classifications sont souvent boiteuses car des pays peuvent être classés dans deux ou trois catégories à la fois!

En fait, ce qui caractérise le sous-développement, du moins ce que les Occidentauxdésignent comme tel, c'est une somme de distorsion qui affligent l'ensemble de ces sociétés; ces pays ressemblent à un malade atteint deplusieurs maladies graves au même moment. L'état de sous-développement peut être représenté dans un schéma:

Le haut du schéma III montre les conditions socio-économiques et politiques qui déterminent le cercle vicieux (bas du schéma III) du sous-développement propre à ces pays. Ce sont donc des causes multiples et particulières qui provoquent la spirale du sous-développement. Pour chacun des pays en développement, ces éléments jouent, à des degrés divers, mais parviennent toujours au même résultat: une régression irréversible du mode de vie.

\section{Deux mythes tenaces}

Le tourisme dans les pays en développement doit faire face à deux mythes tenaces: le premier concerne la mondialisation du tourisme, il suppose que le tourisme international s'est étendu à̀ toute la planète. Le deuxième mythe concerne les impacts négatifs du tourisme sur les pays en développement: il entraînerait, pour ces pays, des catastrophes écologiques et cul turelles.

La mondialisation du tourisme n'est pas pour demain; les arrivées des touristes internationaux ont augmenté de façon importante depuis 1962 mais cette croissance est lente (voir le tableau 1). En 1962,
TABLFAU 2

Les parts de marché des recettes du tourisme international (millions US S) dans les diverses régions du monde de 1988 a 1992 (en \%)

\begin{tabular}{lrrrrr}
\hline Régions & 1988 & 1989 & 1990 & 1991 & 1992 \\
\hline Europe & 53,6 & 51,2 & 53,7 & 51,6 & 52,1 \\
Amériques & 25,2 & 27,4 & 26,7 & 29,1 & 28,0 \\
Afrique & 2,3 & 2,1 & 2,0 & 1,8 & 1,9 \\
Asie & 16,3 & 16,8 & 15,6 & 15,8 & 16,0 \\
Moyen-Orient & 2,5 & 2,5 & 2,0 & 1,7 & 2,0 \\
\hline Total & 100,0 & 100,0 & 100,0 & 100,0 & 100,0 \\
\hline
\end{tabular}

Source: OMT.

\section{TABLEAU 3}

Comparaison des 10 pays les plus pauvres de l'OMT avee les 10 pays les plus riches de l'OCDE pour 3 indicateurs : la fonction touristique par 1000 habitants, la fonction des revenus touristiques par 100 babitants et le nombre de touristes par 100 habitants

\begin{tabular}{|c|c|c|c|}
\hline Pays & $\begin{array}{c}\text { Fonction } \\
\text { touristique } \\
\text { d'hebergement } \\
\text { (pour } 1000 \\
\text { habitants)" }\end{array}$ & $\begin{array}{l}\text { Fonction des } \\
\text { recettes touristiques } \\
\text { (pour } 100 \\
\text { habitants) }\end{array}$ & $\begin{array}{c}\text { Nombre de } \\
\text { touristes } \\
\text { (pour } 100 \\
\text { habitants) }\end{array}$ \\
\hline
\end{tabular}

Les 10 pays les plus pauvres de l'OMT*

$\begin{array}{lccc}\text { Bangladesh } & 0,05 & 6,70 & 0,09 \\ \text { Rwanda } & 0,25 & 53,33 & 0,07 \\ \text { Burundi } & 0,19 & 69,20 & 1,48 \\ \text { Éthiopie } & 0,09 & 41,73 & 0,15 \\ \text { Madagascar } & 0,54 & 303,97 & 0,42 \\ \text { Malawi } & 0,05 & 125,48 & 1,19 \\ \text { Myanmar } & 0,06 & 297,72 & 0,04 \\ \text { Népal } & 0,57 & 534,49 & 1,62 \\ \text { Sierra Leont } & 0,51 & 388,13 & 2,07 \\ \text { Zaïre } & 0,69 & 17,55 & 0,08 \\ & & & \\ \text { Moyenne } & 0,30 & 183,83 & 0,72\end{array}$

Les 10 pays les plus riches de l'OCDE:

$\begin{array}{lccc}\text { Allemagne } & 14,82 & 13630,38 & 18,80 \\ \text { Danemark } & 18,57 & 73191,49 & 214,7 \\ \text { Etats-Unis } & 21,73 & 2112,03 & 17,51 \\ \text { France } & 20,94 & 4370,63 & 104,18 \\ \text { Islande } & 26,83 & 49615,38 & 55,00 \\ \text { Japon } & 3,59 & 2883,08 & 1,69 \\ \text { Luxembourg } & 45,38 & 76923,07 & 204,10 \\ \text { Norvege } & 28,08 & 46037,30 & 55,36 \\ \text { Suède } & 19,15 & 35552,97 & 139,88 \\ \text { Suisse } & 38,57 & 110869,56 & 185,5\end{array}$
Moyenne
23,77
41518.59
99,67

- Les données proviennent de IOMT pour l'année 1991 ou 1992 (selon le pays).

*. La fonction touristique par 1000 habitants : Nombre de places-lit $\times 1000$

Population du pays

w. La fonction des recettes touristiques par 100 habitants (en $\$$ US) :

Recettes du tourisme international du pays $\times 100$

Population du pays
.... Le nombre de touristes par 100 habitants : Nombre de touristes dans le pays $\times 100$ Population du pays 
TABLEAU 4

Thèses et antithèses sur le tourisme dans les pays en développement

Theses

Croissance quasi-infinie du tourisme international

Mondialisation du phénomène touristique

Exploitation des pays pauvres par le tourisme (impacts négatifs)
Antithèses

Croissance oui, mais de plus en plus faible et dépendante du cycle économique

Non - $80 \%$ des échanges se font dans les pays occidentaux ou assimiles.

Ils ne sont pas exploites par le tourisme car il $y$ a tres peu d'echanges avec ces pays

\section{Commentaires}

Le tourisme est une industrie aux mille ramifications maissans unité réelle et sujette à des contre-tendances non seulement Economique (cycle) mais technologique (systemes de communication qui sliminent les distances) et aussi culturelles (effets de mode)

Il s'agit en fait d'une régionalisation: Europe, Amérique du Nord, Asie du Sud Est. Les échanges touristiques ne sont qu'une påle copie des échanges ćconomiques

L'exploitation, s'il y a, dépend beaucoup plus d'une dégradation générale des termes del'échange economiqueavec ces paysqu'au tourisme comme industrie
l'Europe et l'Amérique du Nord concentraient $95 \%$ des échanges touristiques; en 1992, l'Europe et l'Amérique du Nord recevaient encore $81 \%$ des arrivées des touristes internationaux. L'Amérique du Sud, l'Afrique et une grande partiedel'Asie sont pratiquement a bsents de ces échanges internationaux.

L'étude des parts de marché des recettes du tourisme international montre que les pays occidentaux, les trois dragons ariatigues etles Antilles drainaient en 1992 (voir le tableau 2), près de $95 \%$ des recettes globales du tourisme mondial. Ces pays émettent aussi la majorité des dépenses touristiques faites au niveau mondial. Par rapport au commerce mondial, le secteur du tourisme dans les pays en développement est loin de recevoir sa juste part (ces pays représentaient $23,1 \%$ des exportations mondiales en 1990$)$ des recettes mondiales ${ }^{(*)}$.

Le deuxième mythe porte sur lexploitation des pays en développement; dans ces pays, le tourisme aurait des impacts très négatifs sur l'économie, la cultureet l'ensembledes structures sociales. Un des indicateurs utilisés pour démontrer cette exploitation est la part du PNB qui défend directement ou indirectement des recettes touristiques.

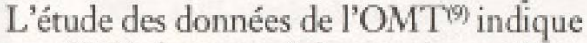
que $90 \%$ des pays où la part du tourisme dans le PNB est supérieure à $10 \%$ sont des petites îles des Antilles, de la Méditerranée ou du Pacifique.
Dans le tableau 3, nous présentons une comparaison des 10 pays les plus pauvres (a partir du revenu moyen par habitant) membres de l'OMT avec les 10 pays les plus riches de l'OCDE (a partir du revenu moyen par habitant). L'indicateur de fonction touristique (selon l'expression de Pierre Defert) $)^{(10)}$ montre les profondes disparités des deux groupes de pays au niveau del'offred'hébergement. Ainsi, le Zaïre(le pays qui a le taux de fonction touristique le plus élevédes pays pauvres) ne possède que $1,5 \%$ de la capacité d'hébergement du pays le plus riche (pour cet indicateur) de I'OCDE (le Luxembourg).

La movenne des recettes touristiques par 100 habitants est de $183,83 \$$ US pour les pays les plus pauvres et de 41518,59 S US pour les pays les plus riches. Nous retrouvons ce mếme énorme décalage par rapport au nombre de touristes pour $100 \mathrm{ha}$ bitants: la moyenne est de 0,72 pour les pays les plus pauvtes et de 99,67 pour les pays les plus riches (11). Il n'y a ni mondialisation ni exploitation pour les pays les plus pauvres: ils ne sont même pas dans le grand jeu du tourisme! Si lexploitation existe, elle doit se faire pour d'autresindustries, à d'autres niveaux.

La majorité des quelques recherches portant sur les impacts culturels du tourisme repose sur des méthodologies qualitatives et porte sur de faibles ćchantillons. Dans la plupart des cas, il est très difficile de dégager clairement ce type d'impact négatif.
Dans le tourisme de masse, les contacts des touristes avec les populations locales sont extrêmementlimités. L'influenceculturelle des médias, des produits culturels et des produits commerciaux (vêtements, automobiles, etc) sont beaucoup plus importants que le tourisme dans les changements culturelsobservés dans les paysen développement. Ceproblèmen'a jamaisétéétudié de façon sérieuse, ce qui fait la vie belle aux idées toutes faites sur cette question!

\section{Quelques paradoxes du développement touristique dans les pays en développement et ailleurs}

Le développement touristique doit quelquefois faire face à certains paradoxes dans les pays en développement. Par exemple, des économistes ont identifié le phénomène de la croisance appawvissante (appelé aussi théorie de Rybczynski). Selon cette theorie: sun nouveau secteur attire les ressources qui quittent le secteur industriels(12); ainsi, la croissance indue d'un secteur particulier (habituellement desressources naturelles) va nuire aux autres secteurs et appauvrir l'ensemble du système économique.

Cette situation se retrouve surtout dans les pays à revenu intermédiaire qui connaissent une assez forte hausse de la croissance touristique (perçue comme une ressource naturelle); ainsi il arrive souvent que le 
développement troprapide du secteur touristique prowoque une certaine forme de désindustrialisation. Ainsi paradoxalement, une croissance touristique trop forte va provoquer une hausse de l'offre et une baisse des prix qui rendra cette croissance nuisible au développement économique global du pays.

D'autres paradoxes apparaissent pour les pays en développement: on exige du secteur touristique de fortes recettes mais en même temps on souhaite qu'il soit quasiinvisible. Il y a un lent ballet d'attraction/ répulsion entre les touristes, l'industrie touristique et les pays en développement.

Les pays occidentaux ne sont pas à court d'attitudes paradoxales face aux pays en développement; les touristes (et les promoteurs du tourisme) s'attendent à :

- un exotisme pondéré;

- une aventure sécuritaire;

- une détente stimulée;

- une nature acclimatée;

- etc.

\section{Thèses et antithèses}

Les liens entre les pays en développement et les pays occidentaux, c'est-à-dire le tourisme, reposent sur une série de malentendus. Tout d'abord, des malentendus psychologiques: dans les pays occidentaux, le tourisme est tout entier simulacre, un construit total et perpétuel élaboré par la dialectique touristes/industrie touristique. Dans les pays en développement, le simulacre doit ếtre dédoublé par l'action de l'industrie touristique locale et les autochtones.

D'autres malentendus sont plus clairs et se basent sur des mythes, des thèses formulées une fois et jamais remises en question. Ces thèses peuvent être illustrées dans un tableau:

Le tourisme tout entier se résume à des formes de thếtrualité - celles-ci exigent, à la fois, d'importants investissements d'infrastructure et de reproduction du système et demande aussi une participation des populations visitées. La quasi-absence des pays du Sud dans le concert touristique mondial s'explique, en grande partie, par l'incapacité à rentabiliser correctement ces lourds investissements des infrastructures et par l'impossibilité pour les visiteurs/' visités de communier dans les mêmes mythes et les mêmes rituels du voyage. En comprenantcela, on saisira quele tourisme ne peut s'exporter comme des voitures ou des machines-outils. $f$

\section{NOTES}

(1) S. Mappa, Cooperation au deveboppement: le refus de latterite, dans Manière de voir, no 22, Le Monde diplomatique, Paris, mai 1994, p. B6.

(2) D'après le celebre livre: E. Dekadt (et autres), Tour isme: passepert pour le dbveloppement? Editions Economica, Paris, 1979

(3) Le tourisme ressemble à la fabrication des saucisses: tout le monde en mange mais personne ne veut savoir comment elles sont produites!

(4) M. Marié, Les terres ot las mots. MeridienKincksieck, Paris, 1989, p. 147.

(5) Voir à ce sujet d. Brasseul, Introduction a l'beonomle du dbveleppement, Armand Colin, Paris, 1989, E. Assidon, Les ther ies economiques du dbveloppement, La Dibouvente, Paris, 1992

(6) A. Hirschman, Stratégie du développement bconomique, Les Editions Ouvrietres, Paris, 1974. p. 84

(7) Voir d ce sujet M. Todaro, Economic Development In the Third World, Longman Inc, N.Y, 1989; et P. Guillaumont, Économie du développement (3 tomes). PUF, Paris, 1965

(B) Voir a ce sujet F. Teulon, La nouvalle dconomie mondiale, PUF, Paris, 1993, D. Auvers, L'deonomie mondiale, La Découverte, Paris, 1987; M. Painelli, Le commer ce international, La Découverte, Paris, 1988

(9) Voir à ce sujet Compendium de statistiques du tourlume 1988-1992, 14 e édition, OMT, Madrid, 1994.

(10) Voir a ce sujet A. Baretje et P. Defert, Aspects bconomiques du tourisme, Berger-Levrault, Paris, 1972, chapitre 3.

(11) II faut noter dans les pays a revenu intermediaire (selon la définition de la Banque Mond ale) que les indicateurs étudiós ont des taux plus tlevés pour les recettes touristiques par 100 habitanits et pour le nombre de touristes par 100 habitants mais sont plus faibles pour les taux de fonction touristique (placestit) par 1000 habitants (movenne autour de 5-6), ce qui semble indiquer que le tourisme ne fait pas encore partie du mode de vie.

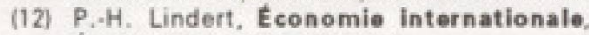
Economica, Paris, 1986, p. 133. 